\section{Bradicardia sinusal sintomática tras inicio de octreotide subcutánea}

\section{Symptomatic sinus bradycardia induced by subcutaneous octreotide}

\section{Sr. Director:}

Presentamos el caso de un paciente varón de 67 años. Como antecedentes personales de interés destacan hipertensión arterial, diabetes mellitus tipo 2, dislipemia, bronquitis crónica, cólicos nefríticos de repetición, cirugía de hemorroides y de hernia umbilical. Tratamiento habitual con insulina aspart, metformina, omeprazol, tiaprida y lorazepam. Sin alergias medicamentosas descritas.

Ingresa en nuestro centro hospitalario para estudio por posible neoplasia de cabeza de páncreas.

Encontrándose hemodinámicamente estable, se inicia pauta con octreotide $100 \mu \mathrm{g} / 8 \mathrm{~h}$ subcutánea.

A las 24 horas presenta bradicardia sinusal a $40 \mathrm{lpm}$ con presión arterial (PA) de 120/60 mmHg, sin sintomatología asociada.

Es valorado por el servicio de cardiología, quienes objetivan en electrocardiograma (ECG) bradicardia sinusal y hemibloqueo de rama anterior izquierda (ya presente en estudios previos), realizan ecocardiograma transtorácico sin alteraciones significativas y colocan Holter de 24 horas.

Presenta deterioro clínico con bradipsiquia e inestabilidad de la marcha; PA, 90/45 mmHg y 35 lpm. Valorada tira de ritmo en planta presenta bradicardia sinusal a 37 lpm y hemibloqueo de rama anterior izquierda descrito (fig. 1). Tras administración de atropina hasta $3 \mathrm{mg}$ persiste bradicardia sintomática. Procedemos a ingreso en la unidad de cuidados intensivos (UCI) e iniciamos perfusión de adrenalina a $10 \mu \mathrm{g} / \mathrm{kg} / \mathrm{min}$, con lo que recupera la frecuencia cardíaca a $60 \mathrm{lpm}$, desaparecen los síntomas descritos y alcanza PA de $120 / 60 \mathrm{mmHg}$. Revisados los fármacos, se suspende la octreotide pautada como posible causa de la sintomatología y alteraciones electrocardiográficas presentes.

Durante las siguientes 12 horas, se suspende paulatinamente la perfusión de adrenalina, y el paciente mantiene una correcta frecuencia cardíaca, presiones arteriales controladas en todo momento, sin episodios arrítmicos significativos. Revisados los datos obtenidos por cardiología del Holter implantado, presenta salva de extrasístole supraventricular aislada.

A las 36 horas del ingreso en $\mathrm{UCl}$ el paciente permanece estable, asintomático y con frecuencias cardíacas en orden (fig. 2) por lo que se le da de alta a planta.

La octreotide es un análogo sintético de la somatostatina con acción inhibitoria de la secreción de la hormona $\mathrm{GH}$ y de diversas hormonas tanto gastrointestinales como pancreáticas. Se encuentra extendido su uso perioperatorio en procedimientos quirúrgicos que afectan a páncreas y vía biliar; tanto en forma profiláctica en complicaciones perioperatorias como en el tratamiento en la fístula biliar postoperatoria.

Existe bibliografía al respecto que acredita efecto bradicardizante de modo significativo ${ }^{1,2}$ como otros artículos en los que no se objetivan diferencias significativas ${ }^{3,4}$. Los mecanismos fisiopatológicos estudiados se relacionan tanto por efecto indirecto en relación con vasoconstricción sistémica ${ }^{5}$ como por efecto directo en relación con la secreción de péptido intestinal vasoactivo y receptores directos de somatostatina localizados en la pared celular de las fibras de conducción miocárdica, lo que produce prolongación del tiempo de conducción auriculoventricular y del periodo refractario del tejido de conducción cardíaca $^{6-8}$.

Para poder establecer el posible efecto secundario de la medicación", primero debemos descartar otros posi-
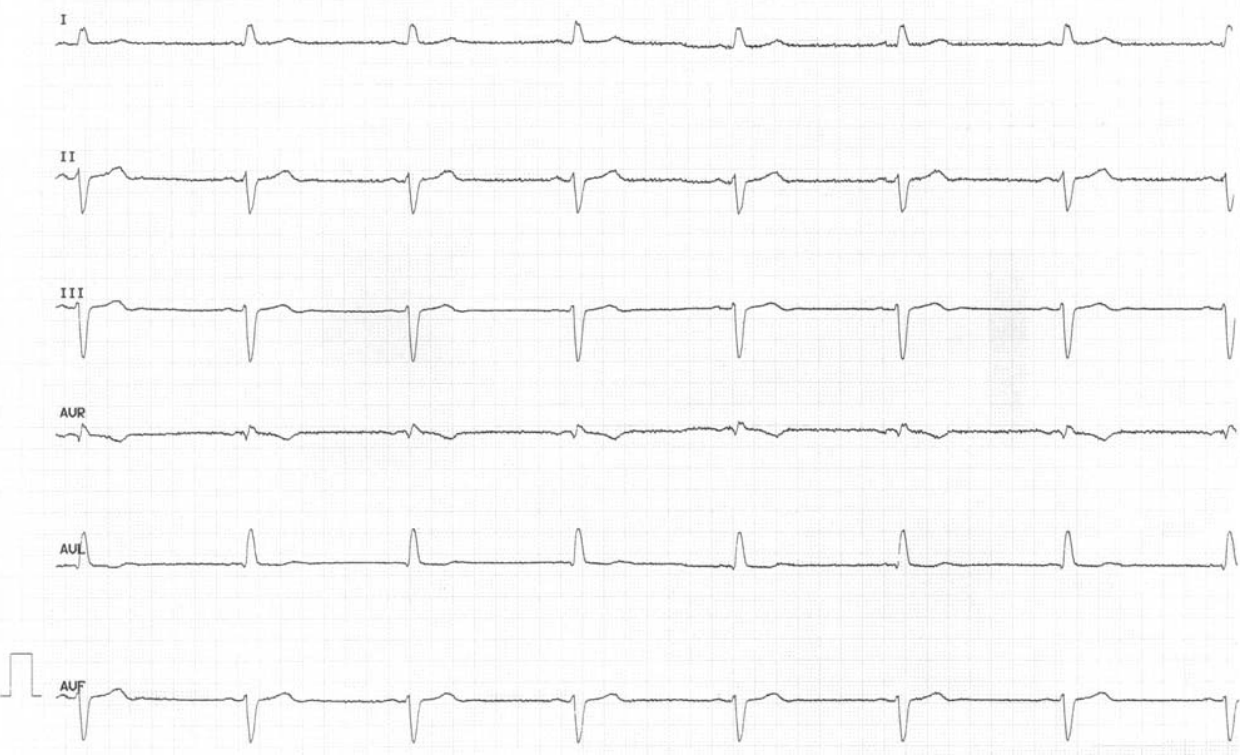

Figura 1 ECG al ingreso. 


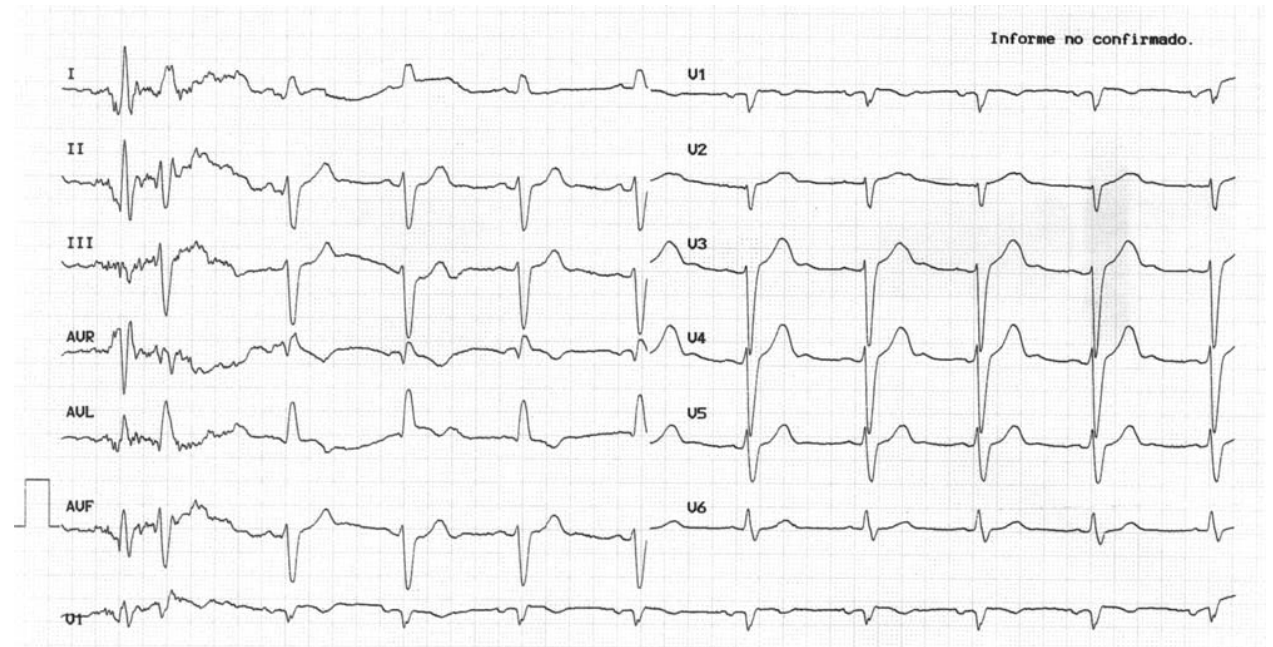

Figura 2 ECG a las 36 horas del ingreso.

bles cuadros que lo justifiquen. En nuestro caso clínico la causa más habitual de bradicardia sinusal se debe a dolor abdominal no controlado, con la consiguiente hiperactividad del sistema parasimpático, el cual no presenta el paciente. Dentro de su tratamiento habitual tampoco se incluyen fármacos con dicho efecto secundario. Incluido en la ficha técnica de la octreotide y en la literatura médica revisada se encuentran los artículos referidos que exponen el potencial efecto secundario de este medicamento. Por último, se objetiva una relación secuencial clara y compatible tanto en la aparición de los síntomas descritos como en la mejoría clínica y electrocardiográfica una vez retirada la medicación descrita del tratamiento habitual.

La importancia del caso radica en que en el perioperatorio debe considerarse este posible efecto secundario incluso en los casos en que se administre de modo subcutáneo.

\section{Bibliografía}

1. Herrington AM, George KW, Moulds CC. Octreotide-induced bradicardia. Pharmacotherapy. 1998;18:413-6.

2. Dilger JA, Rho EH, Qhe FG, Sprung J. Octreotide-induced bradicardia and heart block during surgical resection of a carcinoid tumor. Anesth Analg. 2004;98:318-20.

3. Kinney MAO, Warner ME. Anesthesia for patients with carcinoid tumors. En: Faust RJ, editor. Anesthesiology review. 3. ${ }^{\mathrm{a}}$ ed Nueva York: Churchill Livingstone; 2002. p. 506-7.

4. Sabat M, Guarner C, Soriano G, Bulbena O, Novella MT, Ortiz $\mathrm{J}$, et al. Effect of subcutaneous administration of octreotide on endogenous vasoactive systems and renal function in cirrhotic patients with ascites. Dig Dis Sci. 1998;43:2184-9.
5. McCormick PA, Chin J, Greenslade L, Karatapanis S, Dick R, McIntyre N, et al. Cardiovascular efects of octreotide in patients with cirrhosis. Hepatology. 1995;21:1255-60.

6. Day SM, Gu J, Polak JM, Bloom SR. Somatostatin in human heart an comparasion with guinea pig and rat heart. Br Heart J. 1985;53:153-7.

7. Wiley JW, Uccioli L, Owyang C, Yamada T. Somatostatin stimulates acetylcoline release in the canine heart. Am J Physiol. 1989;257:H483-7.

8. Lamberts SW, Van der Lely AJ, De Herder WW, Hofland LJ. Octreotide. N Engl J Med. 1996;334:246-54.

9. Olivera-González S, De Escalante-Yangüela B, Velilla-Soriano C, Amores-Arriaga B, Martín-Fortea P, Navarro-Aguilar ME. Hepatotoxicidad por metformina. Med Intensiva. 2010;34: 483-7.

M. Cruz Tejedor ${ }^{a, b, *}$ Á.L. Córdoba Sánchez ${ }^{b}$, B. Morató Bellido ${ }^{b}$, Í. Martínez Gil ${ }^{b}$ y J.E. Guerrero Sanz ${ }^{c, d}$

a FEA Medicina Intensiva, Hospital del Sureste, Arganda del Rey, Madrid, España

${ }^{\mathrm{b}}$ Médico especialista en Medicina Intensiva, Servicio de Medicina Intensiva, Hospital Madrid Norte-Sanchinarro, Madrid, España

' Jefe de Servicio de Medicina Intensiva, Grupo Hospital de Madrid, Madrid, España

d Jefe de Servicio de Medicina Intensiva, Hospital General Universitario Gregorio Marañón, Madrid, España

* Autor para correspondencia.

Correo electrónico: manuel_cruz_tejedor@hotmail.com (M. Cruz Tejedor).

doi:10.1016/j.medin.2010.12.005 